\title{
Relationships among Ocular Blood Flow Shown by Laser Speckle Flowgraphy, Retinal Arteriosclerotic Change, and Chorioretinal Circulation Time Obtained by Fluorescein Angiography
}

\author{
Hironori Osamura, Tomoaki Shiba, Takashi Itokawa, Tadashi Matsumoto, and Yuichi Hori \\ Department of Ophthalmology, School of Medicine, Toho University, Tokyo, Japan \\ Correspondence should be addressed to Hironori Osamura; osamura@med.toho-u.ac.jp
}

Received 30 September 2016; Accepted 11 January 2017; Published 26 February 2017

Academic Editor: Lisa Toto

Copyright (c) 2017 Hironori Osamura et al. This is an open access article distributed under the Creative Commons Attribution License, which permits unrestricted use, distribution, and reproduction in any medium, provided the original work is properly cited.

\begin{abstract}
Purpose. To determine the correlations among the mean blur rate (MBR) in the optic nerve head (ONH) shown by laser speckle flowgraphy (LSFG), retinal arteriosclerosis, and the circulation time obtained by fluorescein angiography (FA). Method. We evaluated 118 patients and assessed their time of choroidal flush, arm-to-retina time, and early and late phases of retinal circulation time (RT: sec) obtained by FA. The severity of retinal arteriosclerosis was classified according to the Scheie classification. The MBR values throughout the ONH (MBR-A), in the tissue (MBR-T), and in the vessels (MBR-V) were analyzed. Results. Patients with retinal vein occlusion (RVO) showed prolonged early and late phases of RT compared to other ocular diseases. Single and multiple regression analyses showed that the MBR-V and Scheie classification were significantly associated with both the choroidal flush and arm-to-retina times. The incidences of RVO and MVR-V were significantly associated with the early phase of RT, and the incidences of RVO, MBR-V, Scheie classification, and gender were revealed to be factors independently contributing to the late phase of RT. Conclusion. MBR-V in the $\mathrm{ONH}$ and retinal arteriosclerosis are important contributing factors for the circulation time of each stage obtained by FA.
\end{abstract}

\section{Introduction}

Angiography can be regarded as the classic and standard method for the evaluation of the circulation anywhere in the human body. In the field of ophthalmology, since the development of fluorescein angiography (FA) [1], FA has become the most important method for the evaluation of chorioretinal circulatory disorders. FA gives detailed information about a patient's chorioretinal vascular condition, for example, retinal vein and artery occlusion $[2,3]$. FA can also detect capillary abnormalities such as microaneurysms [4].

FA can reflect the hemodynamics of the chorioretinal circulation in detail. The circulation parameters usually used are the time of choroidal flush, the arm-to-retina circulation time, and the retinal circulation time (RT) [5]. It has been suggested that the choroidal flush and the arm-to-retina times reflect the hemodynamics of the posterior ciliary artery and central retinal artery and that the RT represents the passage time from the first influx of fluorescein dye in the retinal arterioles to its arrival in the retinal venules, passing the retinal microcirculation [5]. Thus, RT can serve as a good indicator of retinal microcirculation.

Several studies have shown that the measurement of RT is reproducible and prolonged in ischemic retinal disease and glaucoma [6-9]. It was also suggested that RT is influenced by retinal arteriosclerosis [10]. However, in rare cases, intravenous fluorescein causes severe side effects $[11,12]$, and thus, it is difficult to perform FA repeatedly for the same patient.

Laser speckle flowgraphy (LSFG) is a noninvasive method for quantifying the ocular blood flow $[13,14]$. This method is based on the changes in the speckle pattern of laser light reflected from the fundus of the eye $[15,16]$. LSFG depends on the movement of erythrocytes in the retina, the choroid and the optic nerve head $(\mathrm{ONH})$, and the mean blur rate (MBR), which is an indicator of blood flow $[17,18]$. In 2008, the LSFG-NAVI ${ }^{\mathrm{TM}}$ system (Softcare Co., Fukuoka, Japan) was approved as a medical apparatus by the Pharmaceuticals and Medical Devices Agency in 
Japan. The MBR is a quantitative index of the blood flow velocity [19-21], and MBR measurements are highly reproducible [22].

Considering these prior findings, we hypothesized that the MBR shown by the LSFG method may reflect each circulation time obtained using FA. The purpose of the present study was thus to determine whether there are significant correlations among the MBR in the $\mathrm{ONH}$ and the circulation times obtained using fluorescein angiography. We also evaluated the relationship between each type of circulation time and the retinal arteriosclerosis by Scheie classification $[23,24]$.

\section{Materials and Methods}

2.1. Subjects. The institutional review board of Toho University Omori Medical Center approved this study. All subjects provided informed consent to participate in this research in accord with the Declaration of Helsinki. We studied 118 consecutive subjects who underwent FA at the Department of Ophthalmology, Toho University Omori Medical Center, between September 1, 2015, and March 1, 2016. Patients who had uveitis, definite atrial fibrillation, or a history of intraocular surgery were excluded.

2.2. FA Measurements. The circulation times shown by FA were obtained by using an IMAGEnet Digital Imaging System $^{\mathrm{TM}}$ (Topcon Co., Tokyo). All patients were evaluated under mydriasis achieved by the administration of eye drops containing $0.5 \%$ tropicamide and $0.5 \%$ phenylephrine hydrochloride (Mydrin-P, Santen Pharmaceutical, Osaka, Japan). After mydriasis was achieved, $500 \mathrm{mg}$ of fluorescein dye (Fluorescite ${ }^{\circledR}$, Alcon Japan, Tokyo) was injected rapidly into a catheterized antecubital vein.

We calculated the following phases in the transit of fluorescein in the fundus: (1) the time of the choroidal flush (sec), (2) the arm-to-retina time (sec), which reflects the early arteriolar phase and was recorded for the appearance of the retinal arterial fluorescence, and (3) the early and late phases of the retinal circulation time (RT: sec). The early phase was measured from the point at which the retinal artery was filled with fluorescence to the time point of the relevant vein laminar flow outset. The late phase was measured from the point at which the retinal artery was filled with fluorescence to the time at which the relevant vein was completely filled. Photographs of the fundus image were obtained at $1 \mathrm{sec}$ intervals until the late phase of RT.

We divided the subjects' diseases into diabetic retinopathy (DR), age-related macular degeneration (AMD), retinal vein occlusion (RVO), and "others."

2.3. The Scheie Classification. The extent and severity of arteriosclerotic change in the retinal arteries were classified according to the following definition [23, 24]. Group 1 was defined as a broadening of the light reflex from the artery, with minimal or no arteriovenous compression. Group 2 was defined as the changes similar to those in stage 1 , but more prominent. In group 3, the arteries have a "copper wire" appearance, the arteriovenous compression is much

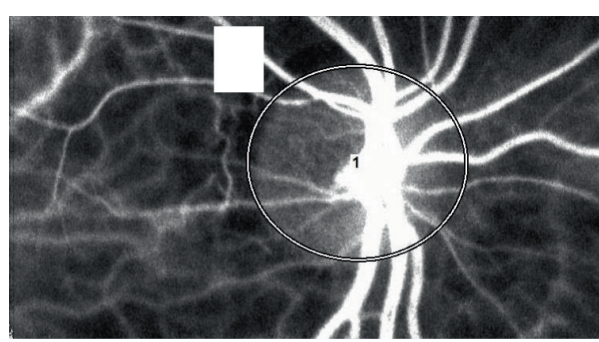

(a)

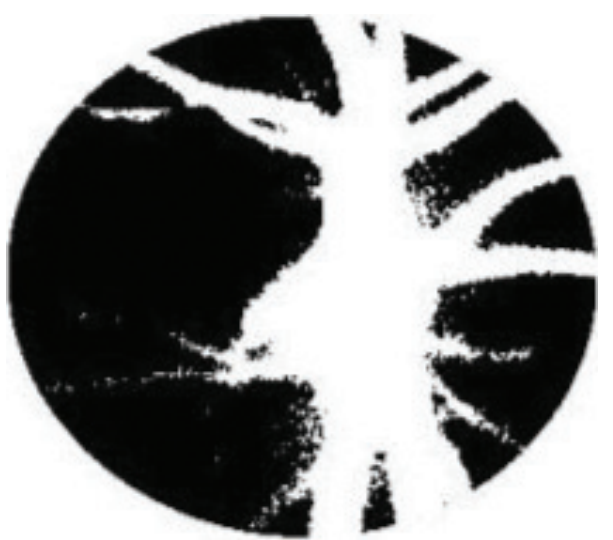

(b)

Figure 1: (a) The margin in the ONH obtained by hand using a round band. (b) The components of vessels and tissues were separated out using the automated definitive threshold.

greater, and serious atherosclerotic changes of the retinal arteries are present. In group 4, the arteries have a "silver wire" appearance, and the arteriovenous crossing changes are the most severe.

The upper temporal quadrants and the first three arterial branchings were analyzed in all patients. The eye fundus examinations were carried out by fundus photography by the same ophthalmologist (H. O.) who had no medical information about the patients. The study eye was chosen as the side opposite the eye used for the FA.

2.4. LSFG Measurements in the ONH. The determination of LSFG from $\mathrm{ONH}$ images has been described in detail $[18,25]$. In the present study, we used the MBR as an indicator of blood flow in the LSFG measurements. We calculated the three parameters of the MBR in the $\mathrm{ONH}$ described below by using the LSFG Analyzer software (v.3.0.47, Softcare). After we had identified the margin of the $\mathrm{ONH}$ by hand using a round band (Figure 1(a)), the software separated out the vessels using the automated definitive threshold (Figure 1(b)) and then analyzed the means of the MBRs in the vessels of the ONH (MBR-V), in the $\mathrm{ONH}$ tissue (MBR-T), and throughout the $\mathrm{ONH}$ (MBR-A) [21, 22,26]. The eye studied was the same side as that used for the FA evaluation. The LSFG measurements were made immediately prior to the FA evaluation and were obtained after the subject rested for $10 \mathrm{~min}$ in a quiet room maintained at $24^{\circ} \mathrm{C}$, with mydriasis. We measured the $\mathrm{ONH}$ three times and used the average for the statistical analyses. 
TABLE 1: Clinical characteristics of the 118 study patients.

\begin{tabular}{lc}
\hline Men : women & $84: 34$ \\
Age (yrs) & $60.4 \pm 15.1$ \\
Diabetes mellitus (\%) & $43(36.4)$ \\
Hypertension (\%) & $50(42.4)$ \\
Anticoagulant agents (\%) & $10(8.5)$ \\
SBP (mmHg) & $142 \pm 20$ \\
DBP (mmHg) & $82 \pm 14$ \\
Heart rate (beat per min) & $78 \pm 14$ \\
\hline Target eye of & $-1.2 \pm 2.8$ \\
\hline Ref (D) & $14.1 \pm 3.1$ \\
IOP (mmHg) & $53.9 \pm 9.7$ \\
OPP (mmHg) & $36(30.5)$ \\
\hline Diseases & $19(16.1)$ \\
\hline DR (\%) & $26(22.0)$ \\
AMD (\%) & $37(31.4)$ \\
RVO (\%) & \\
Others (\%)
\end{tabular}

The data are mean \pm SD or number of patients (\%). SBP: systolic blood pressure, DBP: diastolic blood pressure, Ref: spherical refraction, IOP: intraocular pressure, OPP: ocular perfusion pressure, DR: diabetic retinopathy, AMD: age-related macular degeneration, and RVO: retinal vein occlusion.

2.5. Measurements of Other Parameters. The following parameters were recorded: history of diabetes mellitus, hypertension, and the use of anticoagulant agents. Systolic blood pressure (SBP, $\mathrm{mmHg}$ ), diastolic blood pressure (DBP, $\mathrm{mmHg}$ ), heart rate (beats per min, bpm), mean arterial blood pressure (MABP, $\mathrm{mmHg}$ ), intraocular pressure (IOP, $\mathrm{mmHg}$ ) measured by applanation tonometry, ocular perfusion pressure (OPP, $\mathrm{mmHg}$ ), and spherical refraction (Ref: diopters, D) were assessed with the TONOREF $2^{\mathrm{TM}}$ system (Nidek Co., Aichi, Japan).

Diabetes mellitus was diagnosed in the subjects who were already undergoing treatment for diabetes mellitus, and we diagnosed hypertension if the subject was receiving antihypertensive therapy.

The MABP was determined by the following formula: $\mathrm{DBP}+(\mathrm{SBP}-\mathrm{DBP}) / 3$.

The OPP was defined as (2/3MABP) - IOP.

All parameters were evaluated at the same time as the LSFG measurements.

2.6. Statistical Analysis. The data are presented as the means \pm standard deviation (SD) for the continuous variables. We performed one-way analysis of variance (ANOVA) to determine whether there is a difference in each type of circulation time revealed by FA in the different diseases. We used single regression analysis to determine the relationship between each type of circulation time revealed by FA, the Scheie classification, and the values of MBR-V, MBR-T, and MBR-A in the ONH and other parameters. We used a multiple regression analysis to determine the independent factors for each type of circulation time revealed by FA. $p$ values $<0.05$ were considered significant. The
TABLE 2: Circulation times obtained by FA, arteriosclerosis, and ONH circulation shown by laser speckle flowgraphy.

\begin{tabular}{lc}
\hline Fluorescein angiography (FA) & \\
\hline Time of choroidal flush (sec) & $16.0 \pm 4.4$ \\
Arm-to-retina time (sec) & $18.5 \pm 4.5$ \\
RT: early phase (sec) & $3.4 \pm 1.7$ \\
RT: late phase (sec) & $11.8 \pm 4.0$ \\
\hline Scheie classification & $31(26.3)$ \\
\hline Normal (\%) & $61(51.7)$ \\
Group 1 (\%) & $22(18.6)$ \\
Group 2 (\%) & $4(3.4)$ \\
Group 3 (\%) & $0(0)$ \\
Group 4 (\%) & $38.3 \pm 7.5$ \\
\hline LSFG & $10.9 \pm 2.4$ \\
\hline MBR-V & $20.3 \pm 4.3$ \\
MBR-T & \\
MBR-A &
\end{tabular}

The data are mean \pm SD or number of patients $(\%)(n=118)$.

RT: retinal circulation time, LSFG: laser speckle flowgraphy, and MBR: mean blur rate.

StatView v 5.0 program (SAS Institute, Cary, NC) was used for the statistical analyses.

\section{Results}

Table 1 summarizes the characteristics of the 118 ( 84 men, 34 women) patients. The mean \pm SD age was $60.4 \pm 15.1 \mathrm{yrs}$. Thirty-six patients had DR, 19 patients had AMD, 26 patients had RVO, and 37 patients had other diseases. Table 2 summarizes the circulation time results (i.e., time of choroidal flush, arm-to-retina time, and early and late phases of RT), the retinal arteriosclerosis (Scheie classification), and the MBRs in the ONH obtained by LSFG.

The Scheie classifications were as follows: normal: 31 (26.3\%) patients; group 1: 61 (51.7\%) patients; group 2: 22 (18.6\%) patients; group 3: four (3.4\%) patients; and group 4: $0(0 \%)$ patients. Table 3 shows the FA-measured circulation times for each disease. The early and late phases of RT were significantly longer in the RVO patients than in the patients with DR, AMD, and other diseases (by one-way ANOVA followed by Fisher's PLSD post hoc test, $p=0.005)$, respectively.

Table 4 shows the correlation between each circulation time in FA and the retinal arteriosclerosis by Scheie classification. Time of choroidal flush $(r=0.26, p=0.005)$, arm-to-retina time $(r=0.28, p=0.003)$, and the late phase of RT $(r=0.32, p=0.0004)$ were significantly positively correlated with the progression of Scheie classification.

Table 5 shows the results of the single regression analysis of the relationships among each circulation time in FA and the three sections of MBR in the ONH. The choroidal flush and arm-to-retina times were significantly negatively correlated with MBR-V $(r=-0.37, p<0.0001 ; r=-0.37$, $p<0.0001)$ and MBR-A $(r=-0.27, p=0.003 ; r=-0.25$, $p=0.006$ ), respectively. The early and late phases of RT were significantly negatively correlated with MBR-V 
TABLE 3: Circulation times in each disease as shown by FA.

\begin{tabular}{|c|c|c|c|c|c|}
\hline & DR & AMD & RVO & Others & $p$ value \\
\hline Time of choroidal flush & $16.1 \pm 3.7$ & $15.9 \pm 4.9$ & $15.4 \pm 4.2$ & $16.7 \pm 4.7$ & 0.70 \\
\hline Arm-to-retina time & $18.7 \pm 3.6$ & $18.1 \pm 5.3$ & $17.9 \pm 4.3$ & $19.0 \pm 5.1$ & 0.78 \\
\hline RT: early phase & $3.5 \pm 1.8$ & $3.2 \pm 1.5$ & $4.5 \pm 2.0^{\dagger, \ddagger}$ & $2.9 \pm 1.3$ & 0.005 \\
\hline RT: late phase & $11.4 \pm 3.9$ & $10.8 \pm 3.9$ & $14.4 \pm 4.2^{*}$ & $11.0 \pm 3.3$ & 0.005 \\
\hline
\end{tabular}

The data are mean \pm SD. One-way ANOVA followed by Fisher's PLSD post hoc test. RT: retinal circulation time, DR: diabetic retinopathy, AMD: age-related macular degeneration, and RVO: retinal vein occlusion.

${ }^{\dagger}$ Versus DR, $p<0.05$; " versus AMD and others, $p<0.01$.

${ }^{*}$ Versus DR, AMD, and others, $p<0.01$.

TABLE 4: Correlation between circulation times in FA and ocular arteriosclerosis shown by Scheie classification.

\begin{tabular}{lcc}
\hline Explanatory variable & $r$ value & $p$ value \\
\hline Time of choroidal flush & 0.26 & 0.005 \\
Arm-to-retina time & 0.28 & 0.003 \\
RT: early phase & 0.13 & 0.18 \\
RT: late phase & 0.32 & 0.0004 \\
\hline
\end{tabular}

Objective variables $=$ Scheie classification (normal to group 3 ).

Single regression analysis. RT: retinal circulation time.

$(r=-0.42, p<0.0001 ; r=-0.35, p=0.0001), \mathrm{MBR}-\mathrm{T}$ $(r=-0.34, p=0.0002 ; r=-0.24, p=0.01)$, and MBR-A $(r=-0.39, p<0.0001 ; r=-0.25, p=0.008)$, respectively.

The results of the single regression analysis between each type of circulation time in FA and the subjects' clinical characteristics are shown in Table 6 . Gender $($ men $=1$, women $=0$ ) was correlated significantly with the early phase $(r=0.19, p=0.04)$ and late phase $(r=0.21, p=0.02)$ of RT and tended to be correlated with the choroidal flush $(r=0.17, p=0.07)$ and arm-to-retina times $(r=0.15$, $p=0.10)$ but the correlation did not reach significance. Diabetes mellitus tended to be correlated with the choroidal flush $(r=0.15, p=0.11)$ and the arm-to-retina times $(r=0.18, p=0.06)$. Hypertension was significantly correlated with the choroidal flush $(r=0.20, p=0.03)$ and arm-to-retina times $(r=0.23, p=0.01)$ and tended to be correlated with the early phase $(r=0.16, p=0.09)$ and the late phase $(r=0.17, p=0.07)$ of the RT.

Table 7 shows the results of the multiple regression analysis for factors independently contributing to the time of the choroidal flush and the arm-to-retina time. The MBR-V and Scheie classification were identified as factors contributing independently to both the choroidal flush and arm-to-retina times (time of choroidal flush: MBR-V, standard regression $=-0.32, p=0.0003$; Scheie classification, standard regression $=0.19, p=0.04$; arm-to-retina time: MBR-V, standard regression $=-0.31, p=0.0005$; Scheie classification, standard regression $=0.20, p=0.03$ ).

We also conducted a multiple regression analysis to identify independent contributors to the early and late phases of RT (Table 8). The MBR-V (standard regression $=-0.36$, $p<0.0001)$ and RVO (standard regression $=0.31, p=0.0002$ ) were identified as factors contributing independently to the early phase of RT. RVO (standard regression $=0.32$, $p=0.0001$ ), MBR-V (standard regression $=-0.26, p=0.001$ ), Scheie classification (standard regression $=0.25, p=0.003$ ), and gender $($ men $=1$, women $=0$, standard regression $=0.19$, $p=0.02)$ were all identified as factors contributing independently to the late phase of RT.

\section{Discussion}

Fluorescein angiography is useful for understanding the hemodynamics of the chorioretinal circulation in detail. As noted in the Introduction, intravenous fluorescein can cause severe side effects $[11,12]$, and it may thus be unwise to conduct FA repeatedly for the same patient. Other noninvasive methods for predicting the ocular circulation time are desirable. LSFG is a noninvasive quantitative method for determining the ocular blood flow [13, 14], and the MBR, an LSFG parameter, is a quantitative index of the blood flow velocity [19-21].

A few research groups reported that the MBR-V in the ONH reflected the early phase of RT $[27,28]$, but these studies examined only central RVO cases. It thus remains unclear whether the mean blur rate in the optic nerve head as measured by LSFG reflects the circulation time obtained by FA in different ocular diseases. The relationships between the MBR and circulation times other than those in the early phase of RT also remain to be elucidated.

Our goal in the present study was thus to determine whether there are significant correlations among the $\mathrm{MBR}$ in the $\mathrm{ONH}$, the retinal arteriosclerosis by Scheie classification, and the circulation time obtained by FA, in a variety of ocular diseases of numerous patients. Our results demonstrated that the patients with RVO had prolonged early and late phases of RT compared to the patients with other ocular diseases. Previous studies revealed that the measurement of RT is prolonged in patients with RVO, which is one of the microcirculation disorders [7, 10,29]. Our present findings reconfirmed that the congested retinal venules due to RVO affected the retinal microcirculation shown by RT.

Our single regression analysis showed that the Scheie classification was significantly positively correlated with the time of choroidal flush, the arm-to-retina time, and the late phase of RT. In other words, the time of choroidal flush, arm-to-retina time, and late phase of RT may 
TABLE 5: Correlation between circulation times in FA and MBR values shown by LSFG.

\begin{tabular}{lcccccc}
\hline Explanatory variable & \multicolumn{2}{c}{ MBR-V } & \multicolumn{2}{c}{ MBR-T } & \multicolumn{2}{c}{ MBR-A } \\
\hline Time of choroidal flush & -0.37 & $<0.0001$ & -0.11 & 0.24 & -0.27 \\
Arm-to-retina time & -0.36 & $<0.0001$ & -0.08 & 0.40 & -0.003 \\
RT: early phase & -0.42 & $<0.0001$ & -0.34 & 0.0002 & -0.39 \\
RT: late phase & -0.35 & 0.0001 & -0.24 & 0.01 & -0.25 \\
\hline
\end{tabular}

Single regression analysis. MBR: mean blur rate and RT: retinal circulation time $(n=118)$.

TABLE 6: Results of single regression analysis between the circulation times in FA and clinical characteristics.

\begin{tabular}{|c|c|c|c|c|c|c|c|c|}
\hline \multirow[t]{2}{*}{ Explanatory variable } & \multicolumn{2}{|c|}{$\begin{array}{c}\text { Time of choroidal } \\
\text { flush }\end{array}$} & \multicolumn{2}{|c|}{$\begin{array}{l}\text { Arm-to-retina } \\
\text { time }\end{array}$} & \multicolumn{2}{|c|}{ RT: early phase } & \multicolumn{2}{|c|}{ RT: late phase } \\
\hline & $r$ & $p$ & $r$ & $p$ & $r$ & $p$ & $r$ & $p$ \\
\hline Men $=1$, women $=0$ & 0.17 & 0.07 & 0.15 & 0.10 & 0.19 & 0.04 & 0.21 & 0.02 \\
\hline Age & 0.10 & 0.29 & 0.11 & 0.24 & 0.02 & 0.84 & -0.01 & 0.88 \\
\hline Diabetes mellitus $(+=1,-=0)$ & 0.15 & 0.11 & 0.18 & 0.06 & 0.02 & 0.85 & -0.07 & 0.46 \\
\hline Hypertension $(+=1,-=0)$ & 0.20 & 0.03 & 0.23 & 0.01 & 0.16 & 0.09 & 0.17 & 0.07 \\
\hline Anticoagulant agents $(+=1,-=0)$ & -0.02 & 0.84 & 0.02 & 0.82 & 0.12 & 0.21 & -0.08 & 0.40 \\
\hline SBP & $<-0.01$ & 0.99 & 0.02 & 0.86 & 0.08 & 0.39 & -0.11 & 0.22 \\
\hline DBP & -0.03 & 0.72 & -0.03 & 0.78 & 0.15 & 0.11 & -0.02 & 0.83 \\
\hline Heart rate & -0.10 & 0.30 & -0.07 & 0.45 & 0.04 & 0.64 & $<-0.01$ & 0.99 \\
\hline Ref & 0.04 & 0.70 & 0.04 & 0.68 & -0.03 & 0.77 & -0.06 & 0.52 \\
\hline IOP & 0.03 & 0.76 & -0.01 & 0.91 & -0.01 & 0.95 & 0.04 & 0.65 \\
\hline OPP & -0.03 & 0.74 & -0.01 & 0.95 & 0.13 & 0.15 & -0.08 & 0.39 \\
\hline
\end{tabular}

RT: retinal circulation time; Ref: spherical refraction, IOP: intraocular pressure, and OPP: ocular perfusion pressure $(n=118)$.

be prolonged in parallel with the exacerbation of retinal arteriosclerosis.

The results of the single regression analysis also revealed that the choroidal flush and arm-to-retina times were significantly negatively correlated with the MBR-V and MBR-A and that each phase of the RT was significantly negatively correlated with all sections of the MBR. Among them, the MBR-V was most strongly correlated with all of the circulation times shown by FA. This suggests that the circulation times shown by FA may be prolonged in parallel with the decrease in the MBR-V.

Our multiple regression analysis showed that the MBR-V and retinal arteriosclerosis by Scheie classification were significantly associated with the choroidal flush and arm-to-retina times. The MBR-V, in particular, contributed most strongly to the choroidal flush and arm-toretina times.

Our results also confirmed that the MBR-V and retinal arteriosclerosis may be important components of the hemodynamics of the posterior ciliary artery and the central retinal artery, regardless of the type of ocular diseases.

The multiple regression analysis showed that RVO and MVR-V were significantly associated with the early phase of RT. Moreover, RVO, the MBR-V, the Scheie classification, and gender were also revealed to be factors independently contributing to the late phase of RT, suggesting that RVO and MBR-V may more strongly influence the RT, which is an indicator of retinal microcirculation.

On the other hand, the Scheie classification and gender were also significantly correlated with the late phase of RT. We speculated that the difference in length and weight due to gender differences may affect the late phase of RT. In addition to the MBR-V, retinal arteriosclerosis may be an important factor contributing to the microcirculation shown by RT. Ophthalmologists should pay attention to the importance of Scheie classification not only in light of systemic arteriosclerosis but also concerning ocular microcirculation.

We reported that the secretion levels of vasoactive mediators were significantly correlated with the MBR-V [21]. The MBR-V, representing the vascular component, is considered to be suitable for evaluating intravessel hemodynamics, such as circulation times. Based on our results, we speculate that the combined analysis of the status of the MBR-V and retinal arteriosclerosis may someday be used to predict to some extent the circulation times obtained by FA, which is an invasive method.

Our study has some major limitations. First, there are some problems with the evaluation method of FA. The dye was injected rapidly into a catheterized antecubital vein in all subjects, but there are individual differences in the distance from the arm to the eye, and we did not examine these differences. A reanalysis after adjusting the height, weight, 
TABLE 7: Results of multiple regression analysis for factors independently contributing to the time of the choroidal flush and the arm-to-retina time $(n=118)$.

\begin{tabular}{lccr}
\hline Explanatory variable & \multicolumn{2}{c}{ Time of choroidal flush } & \multicolumn{2}{c}{$\begin{array}{c}\text { Arm-to-retina time } \\
\text { Standard regression }\end{array}$} \\
\hline MBR-V & -0.32 & 0.0003 & -0.31 \\
Scheie classification (normal to group 3) & 0.19 & 0.04 & 0.0005 \\
Men = 1, women $=0$ & 0.08 & 0.34 & 0.06 \\
Diabetes mellitus $(+=1,-=0)$ & 0.09 & 0.31 & 0.03 \\
Hypertension $(+=1,-=0)$ & 0.06 & 0.51 & 0.09 \\
\hline
\end{tabular}

Correlation coefficients: $r=0.46, p<0.0001 ; r=0.47, p<0.0001$.

TABLE 8: Results of multiple regression analysis for factors independently contributing to the retinal circulation time $(n=118)$.

\begin{tabular}{lccr}
\hline Explanatory variable & RT (early phase) & \multicolumn{2}{c}{$\begin{array}{c}\text { RT (late phase) } \\
\text { Standard regression }\end{array}$} \\
\hline RVO $(+=1,-=0)$ & Standard regression & 0.0002 & 0.32 \\
MBR-V & 0.31 & $<0.0001$ & -0.26 \\
Scheie classification (normal to group 3) & -0.36 & & 0.0001 \\
Men $=1$, women $=0$ & & 0.08 & 0.001 \\
Hypertension $(+=1,-=0)$ & 0.14 & 0.31 & 0.02 \\
\hline
\end{tabular}

Correlation coefficients: $r=0.54, p<0.0001 ; r=0.57, p<0.0001$.

RT: retinal circulation time, RVO: retinal vein occlusion, and MBR: mean blur rate.

and distance from the arm to the eye is necessary. Second, an administration of eye drops containing phenylephrine was used for mydriasis for the FA. The phenylephrine might have affected the outcome of the MBR-V measurements.

In addition, we did not evaluate the ocular circulation divided into the individual type (branch or central RVO) or severity of diseases. Further careful validation evaluations are necessary, in accord with the type and severity of various ocular diseases. However, in the present study, a power analysis (linear multiple regression) by $\mathrm{G}^{*}$ Power software (v.3.1.3; developed by Franz Faul, Kiel University, Kiel, Germany) showed that a total sample size of 102 is needed for an effect size $f^{2}$ of 0.15 , an error probability (a) of 0.05 , and a power $(1-b)$ of 0.85 . Therefore, the total sample size of 118 in the present study is a sufficient sample size.

In conclusion, MBR-V representing the vascular component in the optic nerve head as shown by LSFG is an important contributing factor for the circulation time of each stage obtained by fluorescein angiography. Retinal arteriosclerosis may also be an important factor, in the same way.

\section{Competing Interests}

The authors have no financial conflict of interests.

\section{Authors' Contributions}

H. Osamura and T. Shiba were involved in the design and conduct of this study and in the collection, management, analysis, and interpretation of the data. H. Osamura, T. Shiba,
T. Itokawa, T. Matsumoto, and Y. Hori were involved in the preparation, review, or approval of the paper.

\section{References}

[1] H. R. Novotny and D. L. Alvis, "A method of photographing fluorescence in circulating blood in the human retina," Circulation, vol. 24, no. 1, pp. 82-86, 1961.

[2] R. S. Clemett, "Retinal branch vein occlusion. Changes at the site of obstruction," The British Journal of Ophthalmology, vol. 58, no. 5, pp. 548-554, 1974.

[3] J. D. Gass, "A fluorescein angiographic study of macular dysfunction secondary to retinal vascular disease. I. Embolic retinal artery obstruction," Archives of Ophthalmology, vol. 80, no. 5, pp. 535-549, 1968.

[4] E. W. Norton and F. Gutman, "Diabetic retinopathy studied by fluorescein angiography," Ophthalmologica, vol. 150, no. 1, pp. 5-17, 1965.

[5] S. S. Hayreh, "Recent advances in fluorescein fundus angiography," The British Journal of Ophthalmology, vol. 58, no. 4, pp. 391-412, 1974.

[6] B. Bertram, A. Hoberg, S. Wolf, K. Schulte, and M. Reim, "Video fluorescein angiography studies in acute anterior ischemic optic neuropathy," Klinische Monatsblätter für Augenheilkunde, vol. 199, no. 6, pp. 419-423, 1991.

[7] S. Wolf, O. Arend, B. Bertram et al., "Hemodilution therapy in central retinal vein occlusion. One-year results of a prospective randomized study," Graefe's Archive for Clinical and Experimental Ophthalmology, vol. 232, no. 1, pp. 33-39, 1994.

[8] B. Bertram, S. Wolf, S. Fiehofer, K. Schulte, O. Arend, and M. Reim, "Retinal circulation times in diabetes mellitus type 1," The British Journal of Ophthalmology, vol. 75, no. 8, pp. 462-465, 1991. 
[9] N. Plange, M. Kaup, A. Remky, and K. O. Arend, "Prolonged retinal arteriovenous passage time is correlated to ocular perfusion pressure in normal tension glaucoma," Graefe's Archive for Clinical and Experimental Ophthalmology, vol. 246, no. 8, pp. 1147-1152, 2008.

[10] N. Körber, "Measurement of retinal blood flow in various pathological conditions by video fluorescence angiography," Klinische Wochenschrift, vol. 64, no. 19, pp. 950-953, 1986.

[11] M. R. Stein and C. W. Parker, "Reactions following intravenous fluorescein," American Journal of Ophthalmology, vol. 72, no. 5, pp. 861-868, 1971.

[12] L. A. Yannuzzi, K. T. Rohrer, L. J. Tindel et al., "Fluorescein angiography complication survey," Ophthalmology, vol. 93, no. 5, pp. 611-617, 1986.

[13] Y. Tamaki, M. Araie, E. Kawamoto, S. Eguchi, and H. Fujii, "Non-contact, two-dimensional measurement of tissue circulation in choroid and optic nerve head using laser speckle phenomenon," Experimental Eye Research, vol. 60, no. 4, pp. 373-383, 1995.

[14] H. Isono, S. Kishi, Y. Kimura, N. Hagiwara, N. Konishi, and H. Fujii, "Observation of choroidal circulation using index of erythrocytic velocity," Archives of Ophthalmology, vol. 121, no. 2, pp. 225-231, 2003.

[15] H. Fujii, "Visualisation of retinal blood flow by laser speckle flow-graphy," Medical \& Biological Engineering \& Computing, vol. 32, no. 3, pp. 302-304, 1994.

[16] H. Fujii, Laser Speckle Flowgraphy. In Medical Diagnostic Techniques and Procedures, pp. 216-224, Narosa Publishing House, New Delhi, India, 2000.

[17] H. Takahashi, T. Sugiyama, H. Tokushige et al., "Comparison of CCD-equipped laser speckle flowgraphy with hydrogen gas clearance method in the measurement of optic nerve head microcirculation in rabbits," Experimental Eye Research, vol. 108, pp. 10-15, 2013.

[18] T. Sugiyama, "Basic technology and clinical applications of the updated model of laser speckle flowgraphy to ocular diseases," Phonetica, vol. 1, no. 3, pp. 220-234, 2014.

[19] K. Yanagida, T. Iwase, K. Yamamoto et al., "Sex-related differences in ocular blood flow of healthy subjects using laser speckle flowgraphy," Investigative Ophthalmology \& Visual Science, vol. 56, no. 8, pp. 4880-4890, 2015.

[20] N. Aizawa, H. Kunikata, F. Nitta et al., "Age- and sexdependency of laser speckle flowgraphy measurements of optic nerve vessel microcirculation," PLoS One, vol. 11, no. 2, Article ID e0148812, 2016.

[21] T. Shiba, T. Sugiyama, Y. Hori, T. Matsumoto, T. Maeno, and M. Takahashi, "Relationship between plasma levels of vasoactive mediators and optic nerve head circulation shown by laser speckle flowgraphy," Graefe's Archive for Clinical and Experimental Ophthalmology, vol. 254, no. 6, pp. 1033-1039, 2016.

[22] N. Aizawa, Y. Yokoyama, N. Chiba et al., "Reproducibility of retinal circulation measurements obtained using laser speckle flowgraphy-NAVI in patients with glaucoma," Clinical Ophthalmology, vol. 5, pp. 1171-1176, 2011.

[23] H. G. Scheie, "Retinal changes associated with hypertension and arteriosclerosis," The Illinois Medical Journal, vol. 101, no. 3, pp. 126-129, 1952.

[24] H. G. Scheie, "Evaluation of ophthalmoscopic changes of hypertension and arteriolar sclerosis," A.M.A. Archives of Ophthalmology, vol. 49, no. 2, pp. 117-138, 1953.
[25] T. Sugiyama, M. Araie, C. E. Riva, L. Schmetterer, and S. Orgul, "Use of laser speckle flowgraphy in ocular blood flow research," Acta Ophthalmologica, vol. 88, no. 7, pp. 723-729, 2010

[26] M. Ubuka, T. Sugiyama, Y. Onoda, T. Shiba, Y. Hori, and T. Maeno, "Changes in the blood flow of the optic nerve head induced by different concentrations of epinephrine in intravitreal infusion during vitreous surgery," Investigative Ophthalmology \& Visual Science, vol. 55, no. 3, pp. 1625-1629, 2014.

[27] D. Nagasato, Y. Mitamura, K. Semba et al., "Correlation between optic nerve head circulation and visual function before and after anti-VEGF therapy for central retinal vein occlusion: prospective, interventional case series," $B M C$ Ophthalmology, vol. 16, no. 1, 36 pages, 2016.

[28] Y. Yamada, K. Suzuma, M. Matsumoto et al., "Retinal blood flow correlates to aqueous vascular endothelial growth factor in central retinal vein occlusion," Retina, vol. 35 , no. 10 , pp. 2037-2042, 2015.

[29] A. Remky, S. Wolf, M. Hamid et al., "Effect of hemodilution on retinal hemodynamics in retinal branch vein occlusion," Der Ophthalmologe, vol. 91, no. 3, pp. 288-292, 1994. 


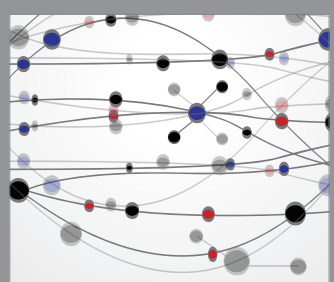

The Scientific World Journal
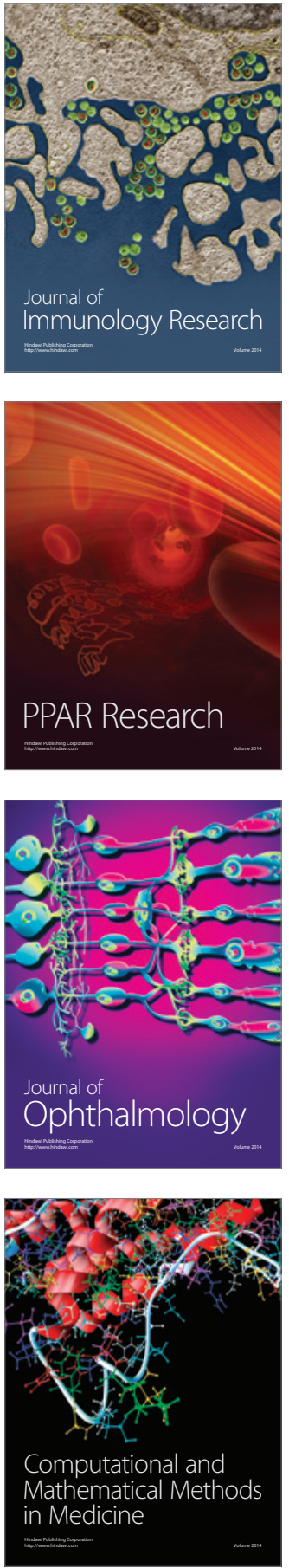

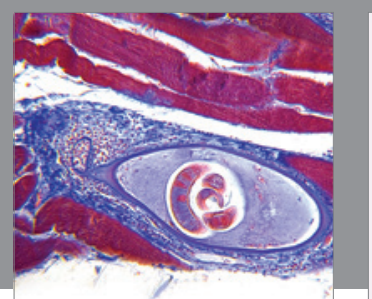

Gastroenterology Research and Practice
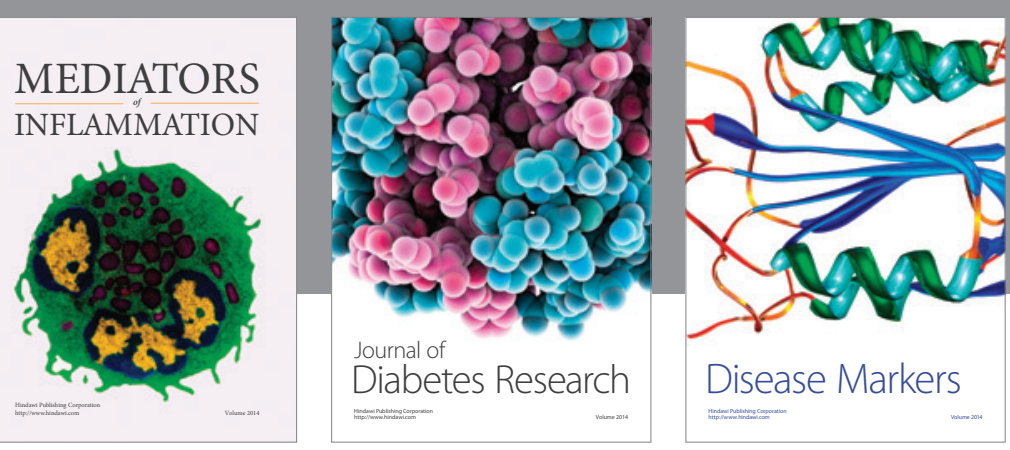

Disease Markers

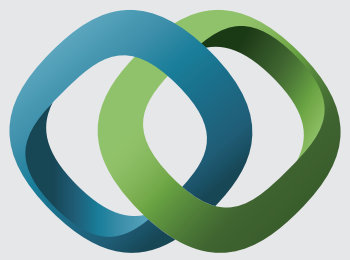

\section{Hindawi}

Submit your manuscripts at

https://www.hindawi.com
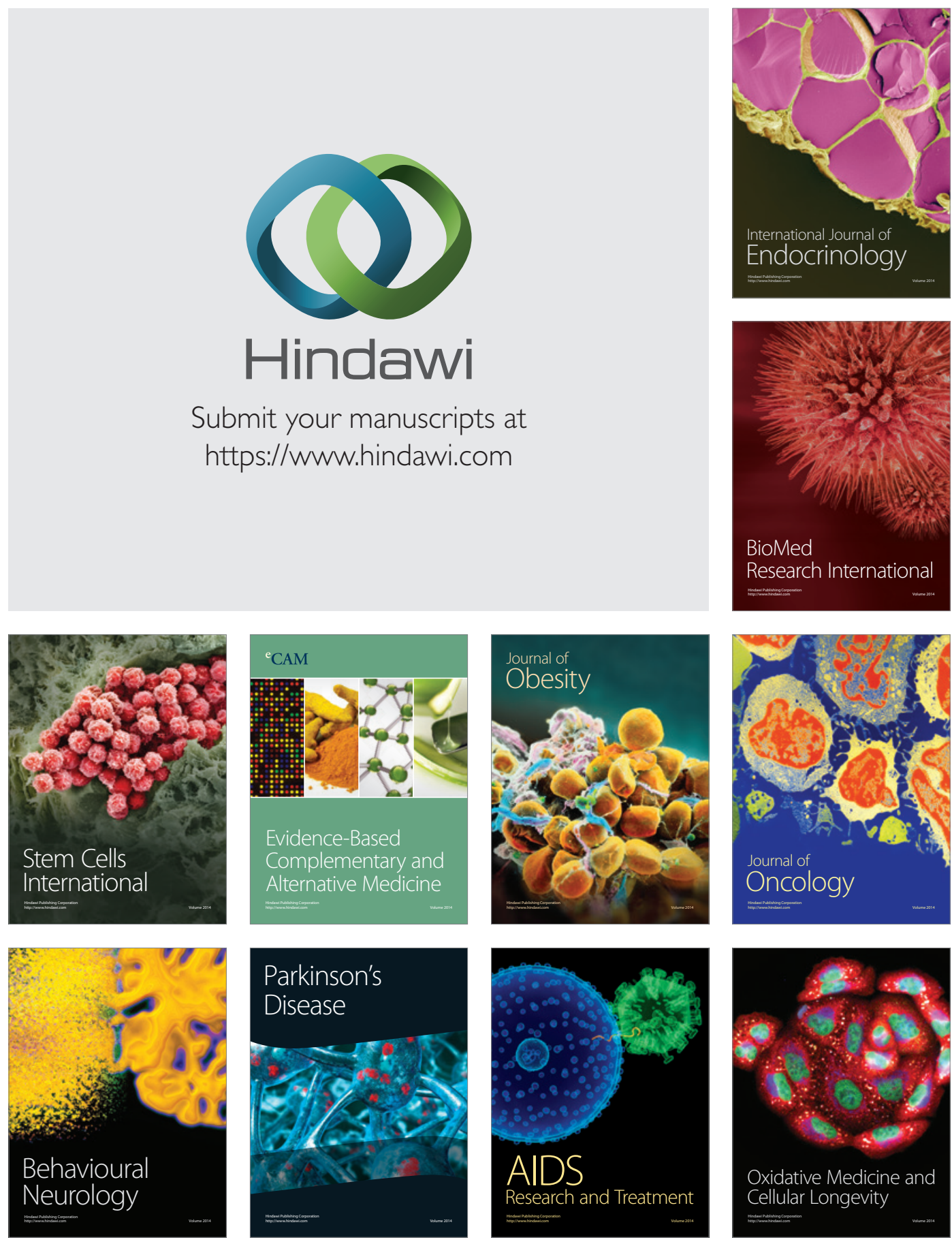